Curr HIV/AIDS Rep. 2013 September ; 10(3): 199-206. doi:10.1007/s11904-013-0168-6.

\title{
Cardiovascular Disease and HIV Infection
}

\author{
Virginia A. Triant, MD, MPH \\ Divisions of Infectious Diseases and General Medicine, Massachusetts General Hospital and \\ Harvard Medical School, Boston, MA, 02114
}

\begin{abstract}
The emergence of chronic disease complications in controlled HIV disease has changed the landscape of HIV clinical care. HIV infection confers an increased cardiovascular disease risk which is thought to be due to a complex interplay of mechanistic factors. While traditional cardiovascular risk factors likely play a role, recent evidence suggests that HIV-associated inflammation and immune activation are important mediators of cardiovascular risk. It is unclear whether established preventative interventions for the general population are applicable to HIVinfected patients, and the need to translate mechanistic knowledge into HIV-specific clinical interventions represents an important priority. Developing strategies to prevent cardiovascular disease in HIV-infected individuals calls for a multidisciplinary approach and represents an opportunity to exert a major public health impact in an at-risk population.
\end{abstract}

\section{Introduction}

As the HIV epidemic has evolved over time, the focus of clinical care has shifted in a way that seldom occurs for a specific disease. In the early stages of the epidemic, opportunistic infections and illnesses related to an immunocompromised state dominated, and a comprehensive body of research and clinical practice developed around these complications. HIV patients on suppressive antiretroviral therapy in the current treatment era confront a new set of complications. These chronic, non-infectious diseases - including cardiovascular disease (CVD) - merit management strategies which differ significantly from those for infectious complications. Moreover, optimal management of cardiovascular disease and other non-infectious complications for an HIV-infected patient may differ from that for an individual without HIV infection. The shifting focus of care for stably treated HIV-infected patients calls for new research into the epidemiology, pathophysiology, prevention, and treatment of chronic disease complications, and the translation of such research into guideline and policy development.

Our knowledge of the increased risk of cardiovascular disease in HIV infection has moved at a rapid pace in recent years, with extensive research on epidemiology and rapidly increasing understanding of mechanism. Considerably less is known about clinical management of CVD for HIV populations. Guidelines developed for the general population may not translate into optimal management in the setting of HIV. The critical next step in the field of HIV and cardiovascular disease will be to translate our understanding of epidemiology and mechanism to the development of HIV-specific clinical management strategies. This review will summarize the state of the field by 1) highlighting recent data on epidemiologic trends in HIV and CVD, focusing on expanded CVD outcomes and specific HIV patient subgroups; 2) describing recent insights into the pathophysiology and

Correspondence and reprints Virginia A. Triant, MD, MPH Massachusetts General Hospital 50 Staniford Street, $9^{\text {th }}$ Floor Boston, MA 02114 Phone: 617-726-5257 Phone: 617-726-2691 vtriant@ partners.org. 
mechanism of HIV-related CVD, including the increasing emphasis on inflammation and immune dysfunction; and 3) discussing the implications of our current knowledge for cardiovascular disease prediction, prevention, and risk factor management for HIV-infected patients.

\section{HIV-associated cardiovascular disease: Advances in epidemiology}

HIV-infected patients confront an increased risk of cardiovascular disease. Multiple observational cohort studies[1-6] have demonstrated elevated rates of acute myocardial infarction (AMI) or coronary heart disease (CHD) in HIV-infected versus control patients, with an approximate 1.5 to 2 -fold increased relative risk. Recent data have reinforced these findings. In a study of more than 27,000 HIV-infected predominantly male patients from the Veterans Aging Cohort Study Virtual Cohort, rates of adjudicated AMI were higher for HIV-infected patients in every age group compared to matched control patients.[7] In multivariate modeling controlling for potentially confounding sociodemographic and cardiovascular covariates, the hazard ratio for AMI associated with HIV status was 1.48, and a significant association between HIV and AMI persisted in an analysis restricted to virologically suppressed patients.[7] A recent meta-analysis that estimated the relative risk of cardiovascular disease among HIV-infected patients found HIV infection to confer a $61 \%$ increased risk of cardiovascular disease endpoints. [8] Moreover, when limited to HIVinfected patients on antiretroviral therapy (ART), the relative risk of CVD remained increased 2-fold compared to HIV-uninfected and 1.5-fold compared to treatment-naïve HIV-infected patients. Inadequate HIV-uninfected control groups in the original studies, unmeasured confounding, and competing risks represent limitations of this analysis.[9] Importantly, both the recent VA study and the meta-analysis found that cardiovascular risk persists even in patients on suppressive antiretroviral therapy, suggesting that CVD is likely to significantly impact the long-term health of this group.

Angiographic presentation, management, and outcomes following an acute cardiovascular event may differ for HIV-infected patients. Several studies have documented clinical and angiographic differences in acute coronary syndrome (ACS) presentation based on HIV status, including higher rates of restenosis,[10] fewer complex lesions,[11] and more frequent presentation with ST-elevation MI versus non-ST-elevation MI or unstable angina[12] in HIV-infected patients versus controls. Studies have differed regarding extent of vessel involvement, with HIV patients having higher rates of single-vessel disease in one study[10] but similar rates of multivessel disease in another.[11] Cardiovascular intervention and procedures have also been shown to differ by HIV status, with lower rates of thrombolysis and anticoagulation, left cardiac catheterization, and coronary artery bypass graft surgery among HIV-infected patients.[13] Finally, HIV infection appears to impact mortality and cardiovascular outcomes following ACS, with one study showing higher inhospital mortality following AMI in adjusted analyses[13] and another showing similar inhospital and 1-year mortality but more subsequent hospitalizations for heart failure among HIV-infected patients.[14] A recent meta-analysis of 11 studies of HIV-infected patients with ACS found higher-than-anticipated rates of in-hospital cardiovascular mortality (8\%) and recurrent AMI (9\%), although control groups were unavailable for comparison.[15]

Although most recent investigation has focused on ischemic heart disease, the effect of HIV on broader cardiovascular outcomes is being increasingly delineated. While early reports of cardiovascular disease in HIV groups focused on cardiomyopathy and cardiovascular manifestations of infections - notably TB pericarditis - patients treated for HIV disease today confront an array of complications spanning the vasculature. HIV-infected patients have been shown to have an increased risk of stroke and cerebrovascular endpoints in comparison to control cohorts, $[16,17]$ and an increasing number of U.S. stroke 
hospitalizations were found to be in the setting of HIV disease between 1997 and 2006.[18] Recently, a study of HIV-infected patients at San Francisco clinic demonstrated a 4.5-fold increase in rates of sudden cardiac death in the HIV group compared to the expected citywide rate.[19] Whether this is related to ischemic CVD versus predisposition to arrhythmias remains unclear. Additional data support a role for HIV in conferring risk for peripheral arterial disease[20] and congestive heart failure,[21] and for more advanced HIV disease to increase risk of incident atrial fibrillation.[22]

Cardiovascular risk may vary within HIV-infected subgroups based on demographic or clinical factors. In several analyses, HIV-infected women appear to be at disproportionately increased CVD risk, with approximately double the relative risk for AMI in HIV-infected women versus men.[4, 6] While absolute CVD rates remain lower for women, HIV infection appears to increase this baseline risk more for women than for men. Although the mechanisms explaining this striking difference have yet to be clearly delineated, differing levels of immune activation by gender at a constant HIV RNA is one potential explanation for the disproportionate impact of HIV on the development of CVD among women.[23]

Co-infections might further modulate CVD risk in HIV disease by altering the degree or nature of the HIV immune response. Hepatitis $\mathrm{C}$ virus (HCV) co-infection has been demonstrated to increase risk of CVD beyond the risk conferred by HIV alone in several studies of CVD outcomes[24, 25] yet a study of AMI rates comparing HCV/HIV co-infected to HIV mono-infected patients did not find a significant difference between the two groups. [26] Several studies focusing on measures of subclinical atherosclerosis have implicated CMV as playing a role in disease progression, $[27,28]$ and a recent study demonstrated HSV-2 seropositivity to be associated with a four-fold increased risk of coronary atherosclerosis as measured by coronary artery calcium (CAC).[29] Additional clinical factors which have been recently shown to confer CVD risk among HIV-infected patients include impaired renal function,[30] vitamin D deficiency,[31] and low serum albumin.[32]

HIV-related immunologic and virologic parameters and disease characteristics have also been demonstrated to predict CVD risk in this population, with associations between 1) low recent CD4 cell count and CVD outcome events in three studies[7, 33, 34]; 2) low nadir CD4 cell count and AMI events[35]; 3) nadir CD4 cell count less than 350 and impaired endothelial function as measured by flow-mediated dilation (FMD) [36]; 4) low CD4 cell count and non-calcified coronary artery plaque[37]; 5) poorer immunologic recovery after ART initiation and increased cardiovascular event rates[38]; and 6) CD4 decline on ART and increased cardiovascular event rates.[39] Increased or detectable HIV RNA has been shown to be significantly associated with several CVD-related endpoints, including AMI,[7, 35] CVD, [40, 41] stroke[17] and non-AIDS event rates.[42] These data suggest that untreated HIV disease or more advanced disease at treatment initiation increases cardiovascular risk, reinforcing the hypothesized role of inflammation and immune activation in mediating risk and further supporting earlier antiretroviral treatment initiation.

\section{Pathophysiology: A shift in understanding}

Expanding knowledge regarding the mechanism of HIV-related CVD has fueled a shift in understanding, with many earlier investigations focusing on the role of traditional CVD risk factors and antiretroviral medications, and more recent inquiry directed towards understanding the roles of immune activation and association inflammation. The true underlying mechanism is likely to reflect a complex interplay of factors partially explained by established mechanistic pathways of cardiovascular disease and partially explained by novel effects related to immunologic sequelae of HIV infection. 
Traditional CVD risk factors, including dyslipidemia, diabetes, smoking and hypertension, have been shown from early in the epidemic to be heightened in HIV groups and have been reviewed previously in detail.[43] Genetic factors have also been explored within HIVinfected patients, in whom an unfavorable genetic risk score based on 23 CHD-associated single-nucleotide polymorphisms (SNPs) conferred a risk similar to that of traditional risk factors.[44] As metabolic abnormalities were observed in association with ART use, investigation turned to possible risk of cardiovascular events related to antiretroviral drugs. Several studies have demonstrated increased CVD risk associated with ART classes[45] or individual medications which are not currently recommended as first-line treatment in the U.S.[46, 47] Newer drugs such as atazanavir do not appear to be implicated in this increased risk,[48] although some newer drugs have yet to be evaluated. While a recent meta-analysis confirmed several of the observed effects from observational studies,[49] other metaanalyses which included data from randomized trials have failed to show associations of individual antiretroviral drugs - abacavir in particular - with AMI.[50, 51]

While plausible that increased CVD risk could be explained by the combined effects of traditional CVD risk factors and cumulative exposure to antiretroviral drugs, the Strategies for Management of Antiretroviral Therapy (SMART) study results prompted a shift in thinking.[52] Although event rates were low, the finding of increased CVD event rates in HIV-infected patients with treatment interruption instigated reconsideration of the balance between toxicity of ART exposure versus that of uncontrolled viremia. These data represent some of the earliest and most robust evidence suggesting a prominent role for the virus and its associated anti-inflammatory effects in mediating CVD risk beyond ART or traditional risk factors.

Recent investigation has consequently focused increasingly on the potential roles of immune activation and inflammation as drivers of cardiovascular disease in HIV, and several recent reviews have been dedicated to understanding this immunopathogenesis.[53-56] Among HIV-infected patients, generalized circulating inflammatory markers have been shown to be associated with ongoing HIV replication,[57] markers of subclinical atherosclerosis, [58] CVD events,[59, 60] and mortality.[61] In a recent analysis of SMART study data, IL-6, hsCRP and D-dimer were shown to be associated with an increased risk of cardiovascular events independent of other CVD risk factors.[62] Studies evaluating predictors of subclinical atherosclerosis as measured by carotid intima-media thickness (cIMT) have differed in terms of the predominant risk factors. While two studies have shown T cell activation to be associated with carotid artery lesions or cIMT, $[63,64]$ another showed cIMT to be associated with traditional CVD risk factors but not with inflammatory markers or HIV viremia.[65] A recent study moved beyond circulating inflammatory markers and assessed arterial inflammation in the coronary vasculature directly. HIV-infected patients were shown to have significantly increased levels of arterial inflammation as assessed by target-to-background ratio in FDG-PET compared to Framingham Risk Score-matched controls patients, and levels similar to those of non-HIV-infected patients with known atherosclerotic disease.[66] Furthermore, HIV-infected patients have been shown to have higher prevalence of vulnerability features of coronary plaque.[67]

Inflammation is heightened in uncontrolled HIV infection, yet evidence suggests that chronic inflammation persists in treated and virologically suppressed HIV-infected patients and may explain elevated CVD rates despite virologic suppression. Multiple studies indicate that levels of inflammatory markers and indicators of immune activation do not fully normalize in the setting of virologic suppression and immune reconstitution with ART.[68, 69] Moreover, several studies focusing on HIV elite controllers - a group that lacks the potential confounding effects of ART - indicate that this persistent inflammation does in fact confer increased CVD risk. A study comparing HIV elite controllers to seronegative 
control patients found increased levels of preclinical atherosclerosis in the elite controllers even after accounting for traditional CVD risk factors.[70] In a recent study utilizing CT angiography, prevalence of atherosclerotic lesions was significantly increased in HIV elite controllers compared with HIV-negative controls.[71]

Most recently, specific pathways of immune activation have been investigated. Monocyte activation has been shown to play a potentially important role in several studies. Notably, a study of activation phenotypes of monocyte subpopulations showed profiles in patients with uncontrolled HIV infection to be similar to those with acute coronary syndrome.[72] Studies linking monocyte activation to CVD outcome measures have largely supported this potential causal pathway. A specific marker of monocyte activation, sCD163, was the only factor significantly associated with vulnerable coronary plaque among HIV-infected patients[67] and was associated with direct arterial inflammation while general inflammatory markers were not.[66] In contrast to these data, a recent study of cIMT found no marker of monocyte activation - including SCD163 and sCD14 - to be associated with the outcome.[64]

\section{Clinical strategies: Moving towards HIV-specific interventions}

While our understanding of epidemiology and pathophysiology is accelerating at a rapid pace, data on CVD management tailored to HIV-infected patients are more limited. Recommendations for management of CVD in the setting of HIV are largely reliant on guidelines and data from the general population.[73, 74] Yet dependence on general population guidelines may not be appropriate for this group of patients, whose risk profile for CVD likely differs from that of the general population. Cardiovascular risk prediction specific to HIV disease has been summarized in detail previously.[75] Although standard CVD risk prediction tools have been applied and compared within HIV cohorts, they have not been formally validated for use in this group of patients. The D:A:D group has developed a risk prediction tool for AMI tailored to HIV-infected patients that incorporates specific antiretroviral medications in addition to common CVD risk factors.[76]

Use of cardiovascular interventions proven beneficial for the general population has been shown to vary, and in many cases be lower than expected, for HIV-infected groups. Patients from the HOPS cohort were assessed in terms of physician compliance to published guidelines for the general population.[77] While the majority was treated pharmacologically for elevated LDL (81-87\%), this was not the case for other parameters (2-11\% treated for low HDL, 56-91\% for hypertriglyceridemia, 46-69\% for hypertension). Moreover, patients in higher cardiovascular risk group were less likely to meet treatment goals. A second recent study showed overall lipid treatment rate to be lower (LDL appropriately treated in $30 \%$ of those at high CVD risk and 50\% of those at moderate risk).[78] Studies of aspirin use have found HIV-infected patients to be prescribed the drug at significantly lower rates than expected.[79, 80] Only about half of HIV-infected patients are treated for diabetes[78] or hypertension.[77, 78] Whether these data reflect suboptimal awareness of CVD preventative measures or the lack of interventions specific for HIV-infected patients is unclear, but recommendations are to treat CVD risk factors at least as aggressively in HIV-infected patients as in the general population.

Smoking is a well-established and intervenable risk factor for cardiovascular disease, with consistently documented heightened rates among HIV-infected patients. In a striking recent study, HIV-infected patients who smoked lost more life years to smoking than to HIV.[81] Within HIV populations, smoking has been linked to an increased cardiovascular event risk, with a population attributable risk for CVD of 25\%.[82] Smoking cessation has been shown to confer cardiovascular benefit for HIV-infected patients, with a reduction in hazard of CVD event risk with longer time since quitting.[83] In recent years smoking cessation has 
increasingly become a recognized priority for HIV-infected patients, $[84,85]$ and several trials have demonstrated promise in designing smoking cessation interventions tailored to HIV-infected patients. A randomized cell phone smoking cessation intervention for HIV patients was shown to be effective, with more than four times increased 7-day abstinence rates.[86] Recently, a large institution-wide smoking cessation training program was developed for HIV clinicians. Compared with other institutions affiliated with an established HIV cohort, patients at the intervention site were more likely to quit smoking and had fewer relapses.[87] Further data will help to inform providers whether cessation methods for the general population can be transferred to HIV groups.

HMG co-A reductase inhibitors (statins) are likely to benefit the HIV population, in light of their anti-inflammatory properties as well as their lipid-lowering capacity, with appropriate consideration of drug interactions. Indeed, statins have been shown to decrease markers of immune activation among HIV-infected patients not on ART[88] and inflammatory markers in treated HIV-infected patients. [89] Data on the effect of statins on clinical outcomes (apart from lipid-lowering effects) among HIV-infected patients are scarce, and no study has specifically investigated the association of statin use with cardiovascular event rates. Allcause mortality was decreased by $67 \%$ with statin use in patients from the Johns Hopkins HIV clinical cohort who achieved virologic suppression after ART initiation.[90] In contrast, a recent study from the Danish nationwide HIV cohort assessed the impact of statin use on all-cause mortality before and after a diagnosis of CVD, CKD, or DM and found a significant association only after the diagnosis of one of the co-morbidities.[91] In a study of a surrogate marker of atherosclerotic disease, treatment with rosuvastatin for 24 months significantly reduced mean cIMT.[92] Ultimately the question of the presence and extent of clinical benefit conferred by statins for HIV populations will best be answered through a clinical trial.

Whether aspirin is effective for all HIV-infected patients or for specific subgroups is an important question that remains as yet unanswered. Aspirin has been shown to be effective in the primary and secondary prevention of AMI in the general population, but no trial has assessed its efficacy for HIV-infected patients. Aspirin's recently demonstrated role in attenuation of platelet activation as well as immune activation in treated HIV disease suggests that it might be an effective preventative measure,[93] yet comparative data on interventions specific to HIV immune dysregulation are lacking. At this time, available guidelines and consensus suggest prescribing aspirin based on the same criteria as those used for the general population.

Novel interventions targeted at inflammation and immune activation are likely to be important components of CVD preventative care among HIV-infected patients, although few have shown clear benefit to date. Hydroxychloroquine failed to reduce $\mathrm{T}$ cell activation among HIV-infected patients and unexpectedly resulted in increases in T cell decline and viral replication.[94] Raltegravir intensification, aimed at further reducing viral replication and associated inflammation, did not improve cardiovascular risk as measured by endothelial function, [95] yet the anti-inflammatory agent pentoxyfylline was shown to reduce circulating markers of endothelial activation.[96] Several conventional treatments have been assessed in novel ways. ACE-inhibitor treatment was shown to reduce inflammatory indices in an HIV group.[97] Vitamin D supplementation, shown to be related to CVD outcomes in HIV-infected patients, was assessed in relation to endothelial function and failed to improve FMD.[98] Finally, low-dose methotrexate, which has been shown to improve inflammatory indices and reduce CVD risk among non-HIV-infected patients, is being investigated in a trial of patients without HIV infection but with a history of CVD and persistent inflammation[99] but has not specifically been studied for HIV-infected groups with respect to cardiovascular prevention. 
An effective potential intervention to prevent HIV-associated CVD is ART itself. Synthesis of epidemiologic data which shows low CD4 and detectable viral load to predict AMI events and mechanistic data invoking inflammation and immune activation in CVD pathogenesis suggests that treating HIV may prevent cardiovascular disease. While individual antiretroviral agents may pose increased risk, the immunologic benefits conferred by ART appear to outweigh this risk. Based on a mathematical model developed to optimize timing of ART initiation with respect to variables including CVD risk - and assuming that ART doubles CVD risk - immediate ART treatment initiation yielded the greatest life expectancy for young patients.[100] Further insight into this critical question will be added by the Strategic Timing of Antiretroviral Treatment (START) trial, designed to assess the risks and benefits, including the role of non-infectious complications, related to the timing of ART initiation.

\section{Future directions and priorities}

Salient gaps exist in our understanding of HIV and cardiovascular disease. While our knowledge has vastly increased compared to a decade ago, critical questions remain unanswered. First, what are the effects of HIV on the heart and vasculature in certain HIV subgroups, including women, children/adolescents and patients in resource-limited settings (RLS)? While summary of data on RLS was beyond the scope of this review, the association of HIV and CVD in this setting remains a research priority, and quantification of specific chronic disease outcome rates will inform clinical care and prevention strategies. Second, what are the long-term consequences of chronic inflammation, and how do these effects interplay with traditional CVD risk factors in the setting of an aging HIV population? Perhaps the relative effects of traditional versus non-traditional risk factors will differ according to age or cumulative viremia/immunosuppression, and interventions will need to be modified accordingly. Third, how does our expanding knowledge on mechanism translate into clinical practice? HIV providers lack evidence-based guidance on how to accurately predict CVD risk and intervene to reduce risk. The development of HIV-specific cardiovascular prevention strategies will vastly improve the care we provide to this at-risk population.

\section{Conclusion}

HIV clinical care is undergoing a significant transition, and our understanding of the intersection between HIV and cardiovascular disease suggests that CVD will disproportionately impact this population's long-term well being. In further expanding and enhancing our knowledge, there are a number of overarching challenges: 1) identifying demographic and clinical HIV subgroups at heightened risk; 2) further delineating CVD mechanism, including interplay of traditional risk factors, ART, and effects of chronic inflammation and immune activation; 3) ensuring aggressive modification of traditional CVD risk factors; 4) translating knowledge of the mediators of CVD to clinically relevant HIV-specific interventions; 5) ascertaining the extent to which vast knowledge of CVD in the general population is relevant to HIV; 6) moving these processed forward in a truly multidisciplinary and coordinated approach, with collaboration between the fields of HIV, cardiology, immunology, epidemiology, and metabolism. Responding to these challenges represents a tremendous opportunity to shape the long term trajectory of clinical care and public health interventions for patients with HIV disease.

\section{References}

1. Currier JS, et al. Coronary heart disease in HIV-infected individuals. Journal of acquired immune deficiency syndromes. 2003; 33(4):506-12. [PubMed: 12869840] 
2. Durand M, et al. Association between HIV infection, antiretroviral therapy, and risk of acute myocardial infarction: a cohort and nested case-control study using Quebec's public health insurance database. Journal of acquired immune deficiency syndromes. 2011; 57(3):245-53. [PubMed: 21499115]

3. Klein D, et al. Do protease inhibitors increase the risk for coronary heart disease in patients with HIV-1 infection? J Acquir Immune Defic Syndr. 2002; 30(5):471-7. [PubMed: 12154337]

4. Lang S, et al. Increased risk of myocardial infarction in HIV-infected patients in France, relative to the general population. AIDS. 2010; 24(8):1228-30. [PubMed: 20400883]

5. Obel N, et al. Ischemic heart disease in HIV-infected and HIV-uninfected individuals: a populationbased cohort study. Clin Infect Dis. 2007; 44(12):1625-31. [PubMed: 17516408]

6 . Triant VA, et al. Increased acute myocardial infarction rates and cardiovascular risk factors among patients with human immunodeficiency virus disease. J Clin Endocrinol Metab. 2007; 92(7):250612. [PubMed: 17456578]

7. Freiberg MS, et al. HIV Infection and the Risk of Acute Myocardial Infarction. JAMA internal medicine. 2013; 173(8):614-22. [PubMed: 23459863] • In this study of a large VA HIV cohort, HIV infection increased risk of myocardial infarction 1.5-fold, adjusting for sociodemographic and cardiovascular confounders. This study corroborates previous data indicating an increased relative risk of myocardial infarction for HIV-infected patients.

8. Islam FM, et al. Relative risk of cardiovascular disease among people living with HIV: a systematic review and meta-analysis. HIV Med. 2012; 13(8):453-68. [PubMed: 22413967]

9. Althoff K, Gange S. A critical epidemiological review of cardiovascular disease risk in HIVinfected adults: the importance of the HIV-uninfected comparison group, confounding, and competing risks. HIV Med. 2013; 14(3):191-192. [PubMed: 23368691]

10. Hsue PY, et al. Clinical features of acute coronary syndromes in patients with human immunodeficiency virus infection. Circulation. 2004; 109(3):316-9. [PubMed: 14718406]

11. Knudsen A, et al. Angiographic features and cardiovascular risk factors in human immunodeficiency virus-infected patients with first-time acute coronary syndrome. Am J Cardiol. 2013; 111(1):63-7. [PubMed: 23040592]

12. Perello R, et al. Clinical presentation of acute coronary syndrome in HIV infected adults: a retrospective analysis of a prospectively collected cohort. European journal of internal medicine. 2011; 22(5):485-8. [PubMed: 21925057]

13. Pearce D, et al. Comparison of in-hospital mortality from acute myocardial infarction in HIV seropositive versus sero-negative individuals. The American journal of cardiology. 2012; 110(8): 1078-84. [PubMed: 22762716]

14. Lorgis L, et al. Outcomes After Acute Myocardial Infarction in HIV-Infected Patients: Analysis of Data From a French Nationwide Hospital Medical Information Database. Circulation. 2013; 127(17):1767-74. [PubMed: 23543004]

15. D'Ascenzo F, et al. Acute coronary syndromes in human immunodeficiency virus patients: a metaanalysis investigating adverse event rates and the role of antiretroviral therapy. Eur Heart J. 2012; 33(7):875-80. [PubMed: 22187508]

16. Rasmussen LD, et al. Risk of cerebrovascular events in persons with and without HIV: a Danish nationwide population-based cohort study. AIDS. 2011; 25(13):1637-46. [PubMed: 21646903]

17. Chow FC, et al. Comparison of ischemic stroke incidence in HIV-infected and non-HIV-infected patients in a US health care system. J Acquir Immune Defic Syndr. 2012; 60(4):351-8. [PubMed: 22580566]

18. Ovbiagele B, Nath A. Increasing incidence of ischemic stroke in patients with HIV infection. Neurology. 2011; 76(5):444-50. [PubMed: 21248273]

19. Tseng ZH, et al. Sudden cardiac death in patients with human immunodeficiency virus infection. $\mathbf{J}$ Am Coll Cardiol. 2012; 59(21):1891-6. [PubMed: 22595409] • HIV-infected patients at a public San Francisco clinic had a striking 4.5-fold increased risk of sudden cardiac death, when compared to the anticipated city-wide rate. As the first study to show such an increase, it highlights the significant impact of CVD-related mortality in HIV groups and underscores the need for prevention strategies. 
20. Ye Y, et al. HIV infection: an independent risk factor of peripheral arterial disease. Journal of acquired immune deficiency syndromes. 2010; 53(2):276-8. [PubMed: 20104124]

21. Butt AA, et al. Risk of heart failure with human immunodeficiency virus in the absence of prior diagnosis of coronary heart disease. Arch Intern Med. 2011; 171(8):737-43. [PubMed: 21518940]

22. Hsu JC, et al. Atrial Fibrillation and Atrial Flutter in HIV-Infected Persons: Incidence, Risk Factors, and Association with Markers of HIV Disease Severity. Journal of the American College of Cardiology. 2013

23. Meier A, et al. Sex differences in the Toll-like receptor-mediated response of plasmacytoid dendritic cells to HIV-1. Nat Med. 2009; 15(8):955-9. [PubMed: 19597505]

24. Bedimo R, et al. Hepatitis $\mathrm{C}$ virus coinfection and the risk of cardiovascular disease among HIVinfected patients. HIV medicine. 2010; 11(7):462-8. [PubMed: 20163481]

25. Freiberg MS, et al. The risk of incident coronary heart disease among veterans with and without HIV and hepatitis C. Circ Cardiovasc Qual Outcomes. 2011; 4(4):425-32. [PubMed: 21712519]

26. Weber R, et al. HBV or HCV coinfections and risk of myocardial infarction in HIV-infected individuals: the D:A:D Cohort Study. Antiviral therapy. 2010; 15(8):1077-86. [PubMed: 21149914]

27. Sacre K, et al. A role for cytomegalovirus-specific CD4+CX3CR1+ T cells and cytomegalovirusinduced T-cell immunopathology in HIV-associated atherosclerosis. AIDS. 2012; 26(7):805-14. [PubMed: 22313962]

28. Parrinello CM, et al. Cytomegalovirus immunoglobulin $\mathrm{G}$ antibody is associated with subclinical carotid artery disease among HIV-infected women. J Infect Dis. 2012; 205(12):1788-96. [PubMed: 22492856]

29. Hechter RC, et al. Herpes simplex virus type 2 (HSV-2) as a coronary atherosclerosis risk factor in HIV-infected men: multicenter AIDS cohort study. Atherosclerosis. 2012; 223(2):433-6. [PubMed: 22472456]

30. Campbell LJ, et al. Renal impairment is associated with coronary heart disease in HIV-positive men. HIV clinical trials. 2012; 13(6):343-9. [PubMed: 23195672]

31. Lai H, et al. Vitamin D deficiency is associated with silent coronary artery disease in cardiovascularly asymptomatic African Americans with HIV infection. Clin Infect Dis. 2012; 54(12):1747-55. [PubMed: 22423137]

32. Lang J, et al. Serum Albumin and Short-Term Risk for Mortality and Cardiovascular Disease among HIV-Infected Veterans. AIDS. 2013

33. Lichtenstein KA, et al. Low CD4+ T cell count is a risk factor for cardiovascular disease events in the HIV outpatient study. Clin Infect Dis. 2010; 51(4):435-47. [PubMed: 20597691]

34. Triant VA, et al. Association of immunologic and virologic factors with myocardial infarction rates in a US healthcare system. Journal of acquired immune deficiency syndromes. 2010; 55(5):615-9. [PubMed: 20827215]

35. Lang S, et al. HIV replication and immune status are independent predictors of the risk of myocardial infarction in HIV-infected individuals. Clinical infectious diseases : an official publication of the Infectious Diseases Society of America. 2012; 55(4):600-7. [PubMed: 22610928]

36. Ho JE, et al. The association of CD4+ T-cell counts and cardiovascular risk in treated HIV disease. AIDS. 2012; 26(9):1115-20. [PubMed: 22382147]

37. Duarte $\mathrm{H}$, et al. Non-calcified coronary plaque volume inversely related to CD4(+) T-cell count in HIV infection. Antivir Ther. 2012; 17(4):763-7. [PubMed: 22293714]

38. van Lelyveld SF, et al. Long-term complications in patients with poor immunological recovery despite virological successful HAART in Dutch ATHENA cohort. AIDS. 2012; 26(4):465-474. [PubMed: 22112603]

39. Helleberg M, et al. CD4 decline is associated with increased risk of cardiovascular disease, cancer and death in virally suppressed HIV patients. Clinical infectious diseases : an official publication of the Infectious Diseases Society of America. 2013

40. Zhang S, et al. Episodes of HIV viremia and the risk of non-AIDS diseases in patients on suppressive antiretroviral therapy. Journal of acquired immune deficiency syndromes. 2012; 60(3): 265-72. [PubMed: 22531756] 
41. Bucher HC, et al. Small dense lipoproteins, apolipoprotein B, and risk of coronary events in HIVinfected patients on antiretroviral therapy: the Swiss HIV Cohort Study. J Acquir Immune Defic Syndr. 2012; 60(2):135-42. [PubMed: 22156913]

42. Lucero C, et al. Rate and predictors of non-AIDS events in a cohort of HIV-infected patients with a CD4 T cell count above 500 cells/mm3. AIDS research and human retroviruses. 2013

43. Grinspoon S, Carr A. Cardiovascular risk and body-fat abnormalities in HIV-infected adults. N Engl J Med. 2005; 352(1):48-62. [PubMed: 15635112]

44. Rotger M, et al. Contribution of genetic background, traditional risk factors and HIV-related factors to coronary artery disease events in HIV-positive persons. Clinical infectious diseases : an official publication of the Infectious Diseases Society of America. 2013

45. Friis-Moller N, et al. Class of antiretroviral drugs and the risk of myocardial infarction. N Engl J Med. 2007; 356(17):1723-35. [PubMed: 17460226]

46. Worm SW, et al. Risk of myocardial infarction in patients with HIV infection exposed to specific individual antiretroviral drugs from the 3 major drug classes: the data collection on adverse events of anti-HIV drugs (D:A:D) study. J Infect Dis. 2010; 201(3):318-30. [PubMed: 20039804]

47. Lang S, et al. Impact of Individual Antiretroviral Drugs on the Risk of Myocardial Infarction in Human Immunodeficiency Virus-Infected Patients: A Case-Control Study Nested Within the French Hospital Database on HIV ANRS Cohort CO4. Arch Intern Med. 2010; 170(14):12281238. [PubMed: 20660842]

48. Monforte A, et al. Atazanavir is not associated with an increased risk of cardio or cerebrovascular disease events. AIDS. 2013; 27(3):407-15. [PubMed: 23291539]

49. Bavinger C, et al. Risk of Cardiovascular Disease from Antiretroviral Therapy for HIV: A Systematic Review. PloS one. 2013; 8(3):e59551. [PubMed: 23555704]

50. Cruciani M, et al. Abacavir use and cardiovascular disease events: a meta-analysis of published and unpublished data. AIDS. 2011; 25(16):1993-2004. [PubMed: 21716077]

51. Ding X, et al. No association of abacavir use with myocardial infarction: findings of an FDA metaanalysis. Journal of acquired immune deficiency syndromes. 2012; 61(4):441-7. [PubMed: 22932321]

52. El-Sadr WM, et al. CD4+ count-guided interruption of antiretroviral treatment. The New England journal of medicine. 2006; 355(22):2283-96. [PubMed: 17135583]

53. Zanni MV, Grinspoon SK. HIV-specific immune dysregulation and atherosclerosis. Current HIV/ AIDS reports. 2012; 9(3):200-5. [PubMed: 22638983]

54. Lo J, Plutzky J. The Biology of Atherosclerosis: General Paradigms and Distinct Pathogenic Mechanisms Among HIV-Infected Patients. J Infect Dis. 2012; 205(Suppl 3):S368-74. [PubMed: 22577210]

55. Deeks SG. HIV infection, inflammation, immunosenescence, and aging. Annu Rev Med. 2011; 62:141-55. [PubMed: 21090961]

56. Hunt PW. HIV and Inflammation: Mechanisms and Consequences. Current HIV/AIDS reports. 2012; 9(2):139-47. [PubMed: 22528766]

57. Armah KA, et al. HIV status, burden of comorbid disease, and biomarkers of inflammation, altered coagulation, and monocyte activation. Clinical infectious diseases : an official publication of the Infectious Diseases Society of America. 2012; 55(1):126-36. [PubMed: 22534147]

58. Hsue PY, et al. Carotid Intima-Media Thickness Progression in HIV-Infected Adults Occurs Preferentially at the Carotid Bifurcation and Is Predicted by Inflammation. Journal of the American Heart Association. 2012; 1(2)

59. Triant VA, Meigs JB, Grinspoon SK. Association of C-reactive protein and HIV infection with acute myocardial infarction. J Acquir Immune Defic Syndr. 2009; 51(3):268-73. [PubMed: 19387353]

60. Ford ES, et al. Traditional risk factors and D-dimer predict incident cardiovascular disease events in chronic HIV infection. Aids. 2010; 24(10):1509-17. [PubMed: 20505494]

61. Tien PC, et al. Inflammation and mortality in HIV-infected adults: analysis of the FRAM study cohort. Journal of acquired immune deficiency syndromes. 2010; 55(3):316-22. [PubMed: 20581689] 
62. Duprez DA, et al. Inflammation, coagulation and cardiovascular disease in HIV-infected individuals. PloS one. 2012; 7(9):e44454. [PubMed: 22970224]

63. Kaplan RC, et al. T cell activation and senescence predict subclinical carotid artery disease in HIVinfected women. The Journal of infectious diseases. 2011; 203(4):452-63. [PubMed: 21220772]

64. Longenecker C, et al. Markers of inflammation and CD8 T-cell activation, but not monocyte activation, are associated with subclinical carotid artery disease in HIV-infected individuals. HIV medicine. 2013

65. Stein JH, et al. Ultrasonographic measures of cardiovascular disease risk in antiretroviral treatment-naive individuals with HIV infection. AIDS. 2012

66. Subramanian S, et al. Arterial inflammation in patients with HIV. JAMA. 2012; 308(4):379-86. [PubMed: 22820791] - Arterial inflammation as assessed by target-to-background ratio in FDGPET was significantly increased in HIV-infected patients compared to matched control patients with similar cardiovascular risk. Monocyte activation marker sCD163 was significantly associated with arterial inflammation, reinforcing the hypothesis that immune activation plays a significant role in HIV-associated CVD.

67. Zanni MV, et al. Increased Coronary Atherosclerotic Plaque Vulnerability by Coronary Computed Tomography Angiography in HIV-Infected Men. AIDS. 2013• In this cross-disciplinary study utilizing advanced cardiovascular imaging techniques, HIV-infected patients had a higher prevalence of vulnerability features of coronary plaque.

68. Mendez-Lagares G, et al. Long-Term Suppressive Combined Antiretroviral Treatment Does Not Normalize the Serum Level of Soluble CD14. The Journal of infectious diseases. 2013; 207(8): 1221-5. [PubMed: 23322858]

69. French MA, et al. Serum immune activation markers are persistently increased in patients with HIV infection after 6 years of antiretroviral therapy despite suppression of viral replication and reconstitution of CD4+ T cells. The Journal of infectious diseases. 2009; 200(8):1212-5. [PubMed: 19728788]

70. Hsue PY, et al. Role of viral replication, antiretroviral therapy, and immunodeficiency in HIVassociated atherosclerosis. Aids. 2009; 23(9):1059-67. [PubMed: 19390417]

71. Pereyra F, et al. Increased coronary atherosclerosis and immune activation in HIV-1 elite controllers. AIDS. 2012; 26(18):2409-12. [PubMed: 23032411]

72. Funderburg NT, et al. Shared monocyte subset phenotypes in HIV-1 infection and in uninfected subjects with acute coronary syndrome. Blood. 2012; 120(23):4599-608. [PubMed: 23065151]

73. Dube MP, et al. Guidelines for the evaluation and management of dyslipidemia in human immunodeficiency virus (HIV)-infected adults receiving antiretroviral therapy: recommendations of the HIV Medical Association of the Infectious Disease Society of America and the Adult AIDS Clinical Trials Group. Clin Infect Dis. 2003; 37(5):613-27. [PubMed: 12942391]

74. Aberg JA, et al. Primary care guidelines for the management of persons infected with human immunodeficiency virus: 2009 update by the HIV medicine Association of the Infectious Diseases Society of America. Clin Infect Dis. 2009; 49(5):651-81. [PubMed: 19640227]

75. D'Agostino RB Sr. Cardiovascular Risk Estimation in 2012: Lessons Learned and Applicability to the HIV Population. J Infect Dis. 2012; 205(Suppl 3):S362-7. [PubMed: 22577209]

76. Friis-Moller N, et al. Predicting the risk of cardiovascular disease in HIV-infected patients: the data collection on adverse effects of anti-HIV drugs study. European journal of cardiovascular prevention and rehabilitation : official journal of the European Society of Cardiology, Working Groups on Epidemiology \& Prevention and Cardiac Rehabilitation and Exercise Physiology. 2010; 17(5):491-501.

77. Lichtenstein KA, et al. Provider compliance with guidelines for management of cardiovascular risk in HIV-infected patients. Preventing chronic disease. 2013; 10:E10. [PubMed: 23347705]

78. Reinsch N, et al. Are HIV patients undertreated? Cardiovascular risk factors in HIV: results of the HIV-HEART study. Eur J Prev Cardiol. 2012; 19(2):267-74. [PubMed: 21450595]

79. Burkholder GA, et al. Underutilization of aspirin for primary prevention of cardiovascular disease among HIV-infected patients. Clinical infectious diseases : an official publication of the Infectious Diseases Society of America. 2012; 55(11):1550-7. [PubMed: 22942209] 
80. Tornero C, Ventura A, Mafe M. Aspirin is indicated for primary prevention of cardiovascular events in HIV-infected patients. J Acquir Immune Defic Syndr. 2010; 54(5):560. [PubMed: 20647827]

81. Helleberg M, et al. Mortality attributable to smoking among HIV-1-infected individuals: a nationwide, population-based cohort study. Clinical infectious diseases : an official publication of the Infectious Diseases Society of America. 2013; 56(5):727-34. [PubMed: 23254417] • HIVinfected patients lost more life years to smoking than to HIV, in this striking recent study. These results underscore the critical importance of smoking cessation in HIV groups and provide a strong message which providers can relay to patients.

82. Lifson AR, et al. Smoking-related health risks among persons with HIV in the Strategies for Management of Antiretroviral Therapy clinical trial. Am J Public Health. 2010; 100(10):1896903. [PubMed: 20724677]

83. Petoumenos $\mathrm{K}$, et al. Rates of cardiovascular disease following smoking cessation in patients with HIV infection: results from the D:A:D study(*). HIV Med. 2011; 12(7):412-21. [PubMed: 21251183]

84. Lifson AR, Lando HA. Smoking and HIV: prevalence, health risks, and cessation strategies. Current HIV/AIDS reports. 2012; 9(3):223-30. [PubMed: 22618079]

85. Niaura R, et al. Interventions to address chronic disease and HIV: strategies to promote smoking cessation among HIV-infected individuals. Current HIV/AIDS reports. 2012; 9(4):375-84. [PubMed: 22972495]

86. Vidrine DJ, et al. Efficacy of cell phone-delivered smoking cessation counseling for persons living with HIV/AIDS: 3-month outcomes. Nicotine Tob Res. 2012; 14(1):106-10. [PubMed: 21669958]

87. Huber M, et al. Outcome of smoking cessation counselling of HIV-positive persons by HIV care physicians. HIV medicine. 2012; 13(7):387-97. [PubMed: 22257025]

88. Ganesan A, et al. High dose atorvastatin decreases cellular markers of immune activation without affecting HIV-1 RNA levels: results of a double-blind randomized placebo controlled clinical trial. J Infect Dis. 2011; 203(6):756-64. [PubMed: 21325137]

89. Calza L, et al. Statin Therapy Decreases Serum Levels of High-Sensitivity C-Reactive Protein and Tumor Necrosis Factor-alpha in HIV-Infected Patients Treated With Ritonavir-Boosted Protease Inhibitors. HIV Clin Trials. 2012; 13(3):153-61. [PubMed: 22592095]

90. Moore RD, Bartlett JG, Gallant JE. Association between use of HMG CoA reductase inhibitors and mortality in HIV-infected patients. PloS one. 2011; 6(7):e21843. [PubMed: 21765919]

91. Rasmussen LD, et al. Statin Therapy and Mortality in HIV-Infected Individuals; A Danish Nationwide Population-Based Cohort Study. PloS one. 2013; 8(3):e52828. [PubMed: 23469159]

92. Calza L, et al. Two-Year Treatment with Rosuvastatin Reduces Carotid Intima-Media Thickness in HIV Type 1-Infected Patients Receiving Highly Active Antiretroviral Therapy with Asymptomatic Atherosclerosis and Moderate Cardiovascular Risk. AIDS Res Hum Retroviruses. 2013; 29(3): 547-56. [PubMed: 23098891]

93. O'Brien M, et al. Aspirin attenuates platelet activation and immune activation in HIV-infected subjects on antiretroviral therapy: A Pilot Study. Journal of acquired immune deficiency syndromes. 2013

94. Paton NI, et al. Effects of hydroxychloroquine on immune activation and disease progression among HIV-infected patients not receiving antiretroviral therapy: a randomized controlled trial. JAMA : the journal of the American Medical Association. 2012; 308(4):353-61. [PubMed: 22820788] - Contrary to the hypothesized result, hydroxychloroquine did not reduce T cell activation among HIV-infected patients in this clinical trial. Moreover, patients randomized to the intervention arm had increased $\mathrm{T}$ cell decline and viral replication. These unexpected results emphasize the challenges of designing interventions to target immune activation in HIV-infected patients.

95. Hatano $\mathrm{H}$, et al. A randomized controlled trial assessing the effects of raltegravir intensification on endothelial function in treated HIV infection. Journal of acquired immune deficiency syndromes. 2012; 61(3):317-25. [PubMed: 22918156]

96. Green LA, et al. Pentoxifylline reduces tumor necrosis factor-alpha and HIV-induced vascular endothelial activation. AIDS Res Hum Retroviruses. 2012; 28(10):1207-15. [PubMed: 22463742] 
97. Baker JV, et al. Angiotensin converting enzyme inhibitor and HMG-CoA reductase inhibitor as adjunct treatment for persons with HIV infection: a feasibility randomized trial. PloS one. 2012; 7(10):e46894. [PubMed: 23082133]

98. Longenecker CT, et al. Vitamin D supplementation and endothelial function in vitamin D deficient HIV-infected patients: a randomized placebo-controlled trial. Antivir Ther. 2012; 17(4):613-21. [PubMed: 22293363]

99. Stein JH, Hsue PY. Inflammation, immune activation, and CVD risk in individuals with HIV infection. JAMA : the journal of the American Medical Association. 2012; 308(4):405-6. [PubMed: 22820794]

100. Negoescu DM, et al. Balancing immunological benefits and cardiovascular risks of antiretroviral therapy: when is immediate treatment optimal? Clinical infectious diseases : an official publication of the Infectious Diseases Society of America. 2012; 55(10):1392-9. [PubMed: 22942203] 